This item was submitted to Loughborough's Research Repository by the author.

Items in Figshare are protected by copyright, with all rights reserved, unless otherwise indicated.

\title{
Secant degree of toric surfaces and delightful planar toric degenerations
}

PLEASE CITE THE PUBLISHED VERSION

http://dx.doi.org/10.1515/advgeom-2012-0023

\section{PUBLISHER}

(c) de Gruyter 2013

\section{VERSION}

VoR (Version of Record)

\section{PUBLISHER STATEMENT}

This work is made available according to the conditions of the Creative Commons Attribution-NonCommercialNoDerivatives 4.0 International (CC BY-NC-ND 4.0) licence. Full details of this licence are available at: https://creativecommons.org/licenses/by-nc-nd/4.0/

\section{LICENCE}

CC BY-NC-ND 4.0

\section{REPOSITORY RECORD}

Postinghel, Elisa. 2019. "Secant Degree of Toric Surfaces and Delightful Planar Toric Degenerations". figshare. https://hdl.handle.net/2134/25237. 


\title{
Secant degree of toric surfaces and delightful planar toric degenerations
}

\author{
Elisa Postinghel* \\ (Communicated by T. Grundhöfer)
}

\begin{abstract}
The $k$-secant degree is studied with a combinatorial approach. A planar toric degeneration of any projective toric surface $X$ corresponds to a regular unimodular triangulation $D$ of the polytope defining $X$. If the secant ideal of the initial ideal of $X$ with respect to $D$ coincides with the initial ideal of the secant ideal of $X$, then $D$ is said to be delightful and the $k$-secant degree of $X$ is easily computed. We establish a lower bound for the 2- and 3-secant degree, by means of the combinatorial geometry of non-delightful triangulations.
\end{abstract}

Key words. Toric varieties, secant varieties, degenerations, polytopes, delightful triangulations. 2010 Mathematics Subject Classification. Primary 14M25; Secondary 14D06, 51N35

\section{Introduction}

There is a long tradition within Algebraic Geometry that studies the dimension and the degree of secant varieties. Let $X \subseteq \mathbb{P}^{r}$ be a projective, irreducible variety of dimension $n$. Its $k$-secant variety $\operatorname{Sec}_{k}(X)$ is defined to be the closure of the union of all the $\mathbb{P}^{k-1}$ 's in $\mathbb{P}^{r}$ meeting $X$ in $k$ independent points. If $\operatorname{Sec}_{k}(X)$ has the expected dimension $k n+k-1$, an interesting question is the following: what is the number $\nu_{k}(X)$ of $k$-secant $\mathbb{P}^{k-1}$ 's to $X$ intersecting a general subspace of codimension $k n+k-1$ in $\mathbb{P}^{r}$ ? This question is open in general.

We approach the problem of computing the number $\nu_{k}$ for toric varieties. The basic strategy was suggested by work of Ciliberto, Dumitrescu and Miranda [5] and Sturmfels and Sullivant [16]. Given a projective toric surface $X$, we perform planar toric degenerations, i.e., we consider regular unimodular triangulations $D$ of the polytope $P$ which defines $X$. The ideal of the central fiber is the monomial initial ideal of the ideal of $X$

* The author was partially supported by Marie-Curie IT Network SAGA, [FP7/2007-2013] grant agreement PITN-GA-2008-214584. 
with respect to a suitable term order $\prec$ which corresponds to the triangulation $D$ (see [15, Theorem 8.3]).

In Section 1 and Section 2 we introduce the objects of our study: convex lattice polytopes, toric varieties, toric degenerations and $k$-secant varieties. In Section 2.2 we introduce the notion of $k$-delightful planar toric degenerations of toric varieties: if the $k$-secant ideal of the initial ideal of $X$ with respect to the degeneration coincides with the initial ideal of the $k$-secant ideal of $X$, then the degeneration is $k$-delightful. Sturmfels and Sullivant proved in [16, Theorem 5.4] that if there exists a triangulation $D$ of $P$ with at least one skew $k$-set, i.e., a subset of $k$ triangles of $D$ that are pairwise disjoint, then $\operatorname{Sec}_{k}(X)$ has the expected dimension and the number of such skew $k$-sets is a lower bound for the number $\nu_{k}(X)$, see Theorem 2.6. If equality holds, then $D$ is $k$-delightful and the flat limit of $\operatorname{Sec}_{k}(X)$ is a union of linear subspaces of dimension $k n+k-1$, hence the initial ideal of the $k$-secant ideal is achieved and in particular the $k$-secant degree is computed. This bound for $\nu_{k}(X)$ is almost never sharp, indeed $k$-delightful degenerations are rare.

In Section 3 we approach the secant degree computation and we improve the lower bound for $\nu_{k}$, for $k=2,3$ (Theorem 3.1). The main tool is taking into account the singularities of the configuration $D$ and explaining how they produce $k$-delightfulness defect. As an application we show how this can be used to achieve information about the initial ideal of the $k$-secant ideal of $X$ with respect to $D$ (Theorem 3.10).

The problem of finding delightful triangulations of polytopes was raised by Sturmfels and Sullivant [16, Section 5]. They explored the existence of such triangulations for Veronese varieties, Segre varieties and rational normal scrolls and they showed that a Gröbner basis can be obtained. In Section 4 we complete the classification of all delightful triangulations for toric surfaces with sectional genus 0 .

Acknowledgments. I would like to thank C. Ciliberto for introducing me to the problem of studying secant varieties while a student of him. I am also deeply grateful to R. Piene for many stimulating discussions during the preparation of this paper and to Wouter Castryck for showing me a good reference for the classification of polytopes. I finally thank the referee for a number of helpful and constructive suggestions in improving the contents of this paper.

\section{Convex lattice polytopes, toric varieties and toric degenerations}

1.1 Census of polytopes in $\mathbb{R}^{2}$ with $g \leq 1$. A lattice point in $\mathbb{R}^{n}$ is a point with integral coordinates. A lattice polytope in $\mathbb{R}^{n}$ is a polytope whose vertices are lattice points. The normalized Ehrhart polynomial of a lattice polytope $P$ in $\mathbb{R}^{n}$ is the numerical function $E_{P}: \mathbb{N} \rightarrow \mathbb{N}, t \mapsto \#\left(t P \cap \mathbb{Z}^{n}\right)$. It is known that $E_{P}$ is a polynomial of degree

$\operatorname{dim}(P): E_{P}=\sum_{i=0}^{\operatorname{dim}(P)} \frac{c_{i}}{i !} t^{i}$. The leading coefficient $c_{\operatorname{dim}(P)}$ is denoted by $\operatorname{Vol}(P)$ and it is called the normalized volume of $P$. If $\operatorname{dim}(P)=n$, we have $\operatorname{Vol}(P)=n ! \cdot V(P)$, where $V(P)$ is the usual Euclidean volume of $P$ (see [15, Chapter 4]). If $\operatorname{dim}(P)=2$, we denote by $\operatorname{Area}(P)$ the normalized volume of $P$. 
Set $n=2$ and denote by $g$ the number of interior lattice points of a plane polytope. In this section we will recall the classification of all convex lattice polytopes in $\mathbb{R}^{2}$ with $g=0$ (see [11, Theorem 4.1.2]) and with $g=1$ (see [11, Theorem 4.2.3]), up to lattice equivalence. An integral unimodular affine transformation in the plane, also known as an equiaffinity, is a linear transformation followed by a translation such that, furthermore, the corresponding matrix has determinant 1 and integral entries. For instance the matrix $\left(\begin{array}{ll}1 & 1 \\ 0 & 1\end{array}\right)$ acts on a polytope by sending the point $(x, y)^{T} \in \mathbb{R}^{2}$ to the point $(x+y, y)^{T} \in \mathbb{R}^{2}$ : the points on the $x$-axis are fixed, while the points on the axis $y=k$ are shifted by $k$ on the right as for example in Figure 1.

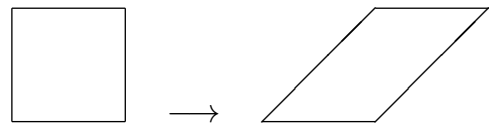

Figure 1. An equiaffinity in the plane.

Normalized area, number of lattice points and convexity of a plane polytope are preserved under these transformations. Two plane polytopes are said to be lattice equivalent if one can be transformed into the other via an equiaffinity.

We refer to [11, Chapter 4] for the proofs of the following results, see also [13].

Theorem 1.1. Any convex lattice polytope $P$ with $\operatorname{dim}(P)=2$ and $g=0$ is lattice equivalent to one of the polytopes in Figure 2.
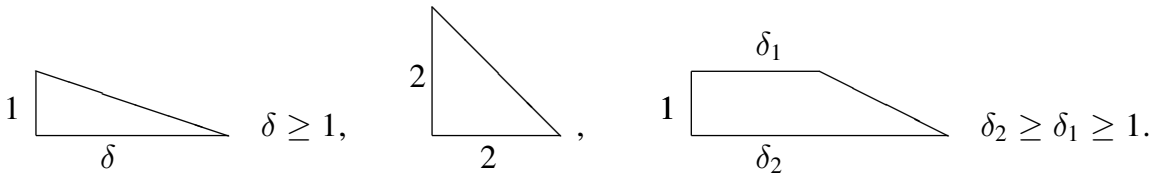

Figure 2. Lattice polygons with $g=0$.

Theorem 1.2. Any convex lattice polytope $P$ with $\operatorname{dim}(P)=2$ and $g=1$ is lattice equivalent to one of the polytopes in Figure 3.

We will use the notation $P^{g}(l, d, m)$ for the lattice equivalence classes of these polytopes, where $l$ is the number of edges (or vertices), $d$ is the normalized area and $m$ is the normalized maximal edge length. The two quadrilaterals with $g=1$ and $l=d=4$ in Figure 3 are not distinguished by this notation, because they both have $m=1$, so we will write $P^{1}(4,4,1)$ for the first one and $\tilde{P}^{1}(4,4,1)$ for the second one.

1.2 Toric varieties via polytopes and toric degenerations. A convex lattice polytope $P \in \mathbb{R}^{n}$ defines a projective toric variety $X_{P}$ of dimension $n$ endowed with an ample line bundle and therefore a morphism into $\mathbb{P}^{r}$, where $r+1$ equals the number of lattice points 

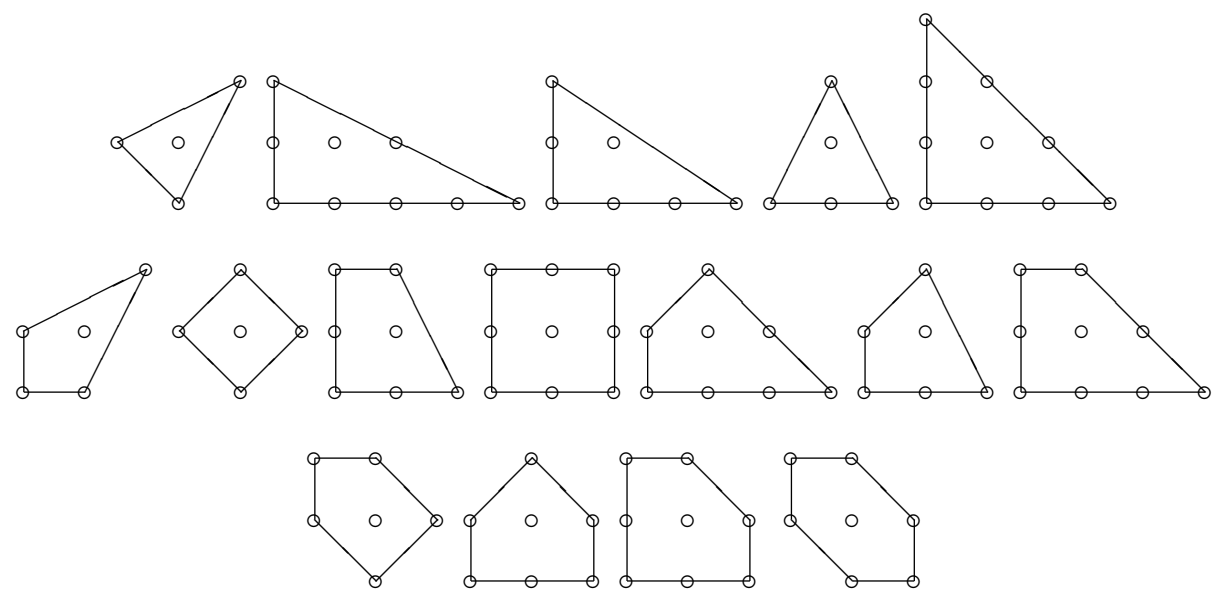

Figure 3. Lattice polygons with $g=1$.

of $P$, as follows. Let $P \cap \mathbb{Z}^{n}=\left\{\underline{m}_{0}, \ldots, \underline{m}_{r}\right\}$ be the set of the lattice points of $P$, with $\underline{m}_{i}=\left(m_{i 1}, \ldots, m_{i n}\right), i=0, \ldots, r$. Consider the monomial map

$$
\begin{aligned}
\Phi_{P}:\left(\mathbb{C}^{*}\right)^{n} & \rightarrow \mathbb{P}^{r} \\
\underline{x} & \mapsto\left[\underline{x}^{\underline{m}}, \ldots, \underline{x}^{\underline{m}} r\right],
\end{aligned}
$$

where $\underline{x}=\left(x_{1}, \ldots, x_{n}\right)$ and $\underline{x}^{\underline{m_{i}}}=x_{1}^{m_{i 1}} \cdots x_{n}^{m_{i n}}$. The projective toric variety $X_{P} \subseteq \mathbb{P}^{r}$ is defined to be the closure of the image of $\Phi_{P}$, see [9, Section 1.5] for more details. The degree of $X_{P}$ equals the normalized volume $\operatorname{Vol}(P)$. Lattice equivalent polygons in $\mathbb{R}^{2}$ define the same toric surface.

A subdivision $D$ of $P$ is a partition of $P$ given by a finite family $\left\{Q_{i}\right\}_{i \in I}$ of convex subpolytopes of maximal dimension such that

- $\bigcup_{i \in I} Q_{i}=P$,

- $Q_{i} \cap Q_{j}$, with $i \neq j$, is either a common face or it is empty.

A subdivision $D$ is said to be regular if there exists a lifting function $F: P \rightarrow \mathbb{R}$, i.e. a piecewise linear positive function satisfying the following requests:

- the $Q_{i}$ 's are the orthogonal projections of the $n$-dimensional faces of the graph polytope $G(F):=\{(x, z) \in P \times \mathbb{R}: 0 \leq z \leq F(x)\}$ of $F$ on $z=0$;

- $F$ is strictly convex.

More details can be found in $[8,10]$. Given a regular subdivision $D$ of $P$, we define a morphism as follows:

$$
\begin{aligned}
\Phi_{D}:\left(\mathbb{C}^{*}\right)^{n} \times \mathbb{C}^{*} & \rightarrow \mathbb{P}^{r} \times \mathbb{C} \\
(\underline{x}, t) & \mapsto\left(\left[t^{F\left(\underline{m}_{0}\right)} \underline{x}^{\underline{m}_{0}}: \cdots: t^{F\left(\underline{m}_{r}\right)} \underline{x}^{\underline{m}_{r}}\right], t\right) .
\end{aligned}
$$


The closure of $\Phi_{D}\left(\left(\mathbb{C}^{*}\right)^{n} \times\{t\}\right)$, for all $t \neq 0$, is a variety $X_{t}$ projectively equivalent to $X_{P}$. The map (1.3) can be extended to a map

$$
\begin{aligned}
& X_{P} \times \mathbb{C}^{*} \rightarrow \mathbb{P}^{r} \times \mathbb{C} \\
& (\underline{x}, t) \quad \mapsto\left(\left[t^{F\left(\underline{m}_{0}\right)} \underline{x}^{\underline{m}_{0}}: \cdots: t^{F\left(\underline{m}_{r}\right)} \underline{x}^{\left.\left.\underline{m}_{r}\right], t\right)}\right.\right.
\end{aligned}
$$

and the flat morphism $\pi_{D}:\left(\left[t^{F\left(\underline{m}_{0}\right)} \underline{x}^{\underline{m}_{0}}: \cdots: t^{F\left(\underline{m}_{r}\right)} \underline{x}^{\underline{m}_{r}}\right], t\right) \mapsto t$ provides a 1dimensional embedded degeneration of $X_{t} \cong X_{P}$ to $X_{0}$ called toric degeneration. The reducible central fiber $X_{0}$ is given by the subdivision $D$ of $P$. The irreducible components of $X_{0}$ are the $X_{Q_{i}}$ 's. The intersections of the components are given by the incidence relations of the corresponding polytopes: if $i \neq j$ and $Q_{i}$ and $Q_{j}$ have a common face $Q_{i} \cap Q_{j}$, then $X_{Q_{i}}$ and $X_{Q_{j}}$ intersect along $X_{Q_{i} \cap Q_{j}}$. We will use the notation $X_{0}=\lim _{D} X_{P}$.

If the subdivision $D$ of $P$ is a regular unimodular triangulation, namely the family $D$ of subpolytopes of $P$ is a simplicial complex whose maximal simplices are unit volume $n$-dimensional tetrahedra $Q_{i}$ 's, then the reducible central fiber $X_{0}$ is a union of linear $n$ dimensional spaces. This leads to the notion of term order. In fact, if $\mathcal{I} \subseteq \mathbb{C}\left[x_{0}, \ldots, x_{r}\right]$ is the homogeneous prime ideal defining $X_{P}$ and $\prec$ is any term order in $\mathbb{C}\left[x_{0}, \ldots, x_{r}\right]$, then the radical of the initial ideal $\operatorname{in}_{\prec}(\mathcal{I})$ is a squarefree monomial ideal whose corresponding simplicial complex $\Delta_{\prec}(\mathcal{I})$ is a regular triangulation of the polytope $P$ defining $X$. Conversely any regular triangulation of $P$ is of the form $\Delta_{\prec}(\mathcal{I})$, for some $\prec$, see [15, Chapter 8].

If $n=2$ and $D$ is a regular unimodular triangulation of $P$, we will say that $\pi_{D}$ is a planar toric degeneration of $X_{P}$.

\section{Secant varieties of toric varieties: a combinatorial approach}

Let $X \subset \mathbb{P}^{r}$ be an irreducible, non-degenerate, projective variety of dimension $n$. Fix an integer $k \geq 2$ and consider the $k$-th symmetric product $\operatorname{Sym}^{k}(X)$. We define the abstract $k$-secant variety of $X, S_{X}^{k} \subseteq \operatorname{Sym}^{k}(X) \times \mathbb{P}^{r}$, as the Zariski closure of the set

$$
\left\{\left(\left(x_{1}, \ldots, x_{k}\right), z\right) \in \operatorname{Sym}^{k}(X) \times \mathbb{P}^{r}: \operatorname{dim}(\pi)=k-1 \text { and } z \in \pi\right\}
$$

where $\pi=\left\langle x_{1}, \ldots, x_{k}\right\rangle$. It is irreducible of dimension $k n+k-1$. Consider the projection $p_{X}^{k}$ on the second factor and define the $k$-secant variety of $X, \operatorname{Sec}_{k}(X):=p_{X}^{k}\left(S_{X}^{k}\right)$, as the image of $S_{X}^{k}$ in $\mathbb{P}^{r}$. It is an irreducible algebraic variety of dimension $\operatorname{dim}\left(\operatorname{Sec}_{k}(X)\right) \leq$ $\min \{k n+k-1, r\}$. The right hand side is called the expected dimension of $\operatorname{Sec}_{k}(X)$. If strict inequality holds then $X$ is said to be $k$-defective, if equality holds then $X$ is said to be non- $k$-defective.

The general fiber of $p_{X}^{k}$ is pure of dimension $k n+k-1-\operatorname{dim}\left(\operatorname{Sec}_{k}(X)\right)$. Denote by $\mu_{k}(X)$ the number of irreducible components. If $\operatorname{dim}\left(\operatorname{Sec}_{k}(X)\right)=k n+k-1 \leq r$, then $p_{X}^{k}$ is generically finite and the number $\mu_{k}(X)=\operatorname{deg}\left(p_{X}^{k}\right)$ is called the $k$-secant order of $X$ (see [4]), i.e., $\mu_{k}(X)$ counts how many $k$-secant $\mathbb{P}^{k-1}$ 's to $X$ pass through the general point of $\operatorname{Sec}_{k}(X)$. This number is equal to one unless $X$ is $k$-weakly defective. 
The weakly defective surfaces are classified in [3]. Let now $L$ be a general linear subspace of $\mathbb{P}^{r}$ of codimension $k n+k-1: X$ has

$$
\nu_{k}(X)=\mu_{k}(X) \cdot \operatorname{deg}\left(\operatorname{Sec}_{k}(X)\right)
$$

$k$-secant $\mathbb{P}^{k-1}$ 's meeting $L$. Notice that if $\nu_{k}(X)=1$, then $\operatorname{Sec}_{k}(X)=\mathbb{P}^{r}$ and $\mu_{k}(X)=$ 1 which means that for a general points of $\operatorname{Sec}_{k}(X)$ there is a unique $k$-secant $\mathbb{P}^{k-1}$.

In this paper we present a combinatorial framework for the computation of the number $\nu_{k}(X)$, for any toric surface $X$.

2.1 The $k$-secant degree of toric surfaces with $g \leq 1$. In this section we will describe the secant varieties of the toric surfaces defined by the polytopes of Theorem 1.1 and Theorem 1.2. They are all minimal $k$-secant degree surfaces, $\mathcal{M}^{k}$-surfaces (see [6]), i.e. $\operatorname{deg}\left(\operatorname{Sec}_{k}(X)\right)=\left(\begin{array}{c}r-\operatorname{dim}\left(\operatorname{Sec}_{k}(X)\right)+k \\ k\end{array}\right)$.

2.1.1 $g=\mathbf{0}$. The 2-Veronese embedding $V_{2}$ of $\mathbb{P}^{2}$ into $\mathbb{P}^{5}$ is described by the triangle $P^{0}(3,4,2)$. The 2-secant variety $\operatorname{Sec}_{2}\left(V_{2}\right)$ is a well-known hypersurface of degree 3 . Moreover $\operatorname{Sec}_{k}\left(V_{2}\right)=\mathbb{P}^{5}, k \geq 3$.

Let $S=S(0, i) \subseteq \mathbb{P}^{i+1}$ be the rational normal cone defined by the triangles $P^{0}(3, i$, $i$ ). We have $\operatorname{dim}\left(\operatorname{Sec}_{k}(S)\right)=\min \{i+1,2 k\}$ (see [6, Proposition 1.14]). In particular $\operatorname{Sec}_{k}(S)=\mathbb{P}^{i+1}$ if $i \leq 3 k-3$, while $S$ is $k$-defective if $i \geq 3 k-2$. Furthermore $S$ is an $\mathcal{M}^{k}$-surface if $i+1 \geq 2 k$, [6, Claim 5.2].

Let $S=S(\delta, \delta+i) \subseteq \mathbb{P}^{2 \delta+i+1}, i \geq 0$, be the rational normal surface scroll whose polygon is the trapezium $P^{0}(4,2 \delta+i, \delta+1)$. If $k-1 \leq \delta$ and $3 k-1 \leq 2 \delta+i+1$ then $S$ is a non- $k$-defective $\mathcal{M}^{k}$-surface. In particular $\operatorname{deg}\left(\operatorname{Sec}_{k}(S)\right)=\left(\begin{array}{c}2 \delta+i-2 k+2 \\ k\end{array}\right)$ and $\mu_{k}(S)=1, k \geq 2$.

A determinantal presentation for the ideals of their $k$-secant varieties is known, see [1, Proposition 2.2].

2.1.2 $\boldsymbol{g}=1$. The $k$-secant varieties of the cubic toric surface in $\mathbb{P}^{3}$ defined by $P^{1}(3,3$, 1) fill up $\mathbb{P}^{3}$, for each $k \geq 2$. The same holds for the quartic toric surfaces in $\mathbb{P}^{4}$ defined by $P^{1}(3,4,2), P^{1}(4,4,1)$ and $\tilde{P}^{1}(4,4,1)$.

Let $V_{3}$ be the 3 -Veronese embedding of $\mathbb{P}^{2}$ into $\mathbb{P}^{9}$, described by the triangle $P^{1}(3,9$, $3)$. It is a non- $k$-defective $\mathcal{M}^{k}$-surface for $k=2,3$. In particular $\operatorname{Sec}_{2}\left(V_{3}\right)$ has dimension 5 and degree 15, while $\operatorname{Sec}_{3}\left(V_{3}\right)$ has dimension 8 and degree 4. Moreover $\operatorname{Sec}_{k}\left(V_{3}\right)=\mathbb{P}^{9}, k \geq 4$. The $j$-internal projections of $V_{3}$, i.e. the surfaces obtained from $V_{3}$ as projections from $j$ general points on it, $1 \leq j \leq 4$, are del Pezzo surfaces of degree $9-j$ in $\mathbb{P}^{9-j}$. Consider in particular the ones defined by the subpolytopes of $P^{1}(3,9,3)$ with $g=1: P^{1}(4,8,3), P^{1}(4,7,3), P^{1}(5,7,2), P^{1}(3,6,3), P^{1}(4,6,2)$, $P^{1}(5,6,2), P^{1}(6,6,1), P^{1}(4,6,2), P^{1}(5,5,1)$. For $k=2$, we have $\operatorname{dim}\left(\operatorname{Sec}_{2}(X)\right)=5$ and $\nu_{2}(X)=\left(\begin{array}{c}d-3 \\ 2\end{array}\right)$. For $k \geq 3, \operatorname{Sec}_{3}(X)=\mathbb{P}^{9-j}$. All of them and their $k$-secant varieties have nice determinantal presentations, for an overview see [2, 12].

Let now $X, Y \subseteq \mathbb{P}^{8}$ be the 2 -Veronese embedding of the smooth quadric $\mathbb{P}^{1} \times \mathbb{P}^{1} \subseteq \mathbb{P}^{3}$ and of the cone in $\mathbb{P}^{3}$ over a rational normal conic, respectively given by $P^{1}(4,8,2)$ and $P^{1}(3,8,4)$. The surfaces $X$ and $Y$ both have 2-secant variety of dimension 5 and degree 10 and 3-secant variety of dimension 7 and degree 4, see [6, Theorem 9.1]. 
2.2 $k$-delightful planar toric degenerations. In this section we will introduce the notion of $k$-secant ideals of a given ideal and we will study flat deformations to their initial ideals.

Let $\mathcal{I}$ be any ideal in the polynomial ring $K\left[x_{0}, \ldots, x_{r}\right]$. The secant $\mathcal{I}^{\{2\}}=\mathcal{I} * \mathcal{I}$ of $\mathcal{I}$ is an ideal in $K\left[x_{0}, \ldots, x_{r}\right]$ defined in the following way. Consider the polynomial $\operatorname{ring} K[\underline{x}, \underline{y}, \underline{z}]=K\left[x_{0}, \ldots, x_{r}, y_{0}, \ldots, y_{r}, z_{0}, \ldots, z_{r}\right]$. Let $\mathcal{I}(y)$ and $\mathcal{I}(\underline{z})$ be the ideals obtained as images of $\mathcal{I}$ in $K[\underline{x}, \underline{y}, \underline{z}]$ via the maps $x_{i} \mapsto y_{i}$ and $\bar{x}_{i} \mapsto z_{i}$, for $i=0, \ldots, r$. Then $\mathcal{I}^{\{2\}}$ is defined as the elimination ideal $\left(\mathcal{I}(\underline{y})+\mathcal{I}(\underline{z})+\left\langle y_{i}+z_{i}-x_{i}: 0 \leq i \leq\right.\right.$ $r\rangle) \cap K\left[x_{0}, \ldots, x_{r}\right]$. The $k$-secant ideals $\mathcal{I}^{\{k\}}=\mathcal{I} * \cdots * \mathcal{I}, k \geq 3$, of $\mathcal{I}$ are similarly defined.

Remark 2.1. For homogeneous prime ideals, the $k$-secant ideals represent the prime ideals of the $k$-secant varieties of irreducible projective varieties.

Let now $\prec$ be any term order. The initial ideal of the $k$-secant ideal $\mathcal{I}^{\{k\}}$ of $\mathcal{I}$ is contained in the $k$-secant of the initial ideal of $\mathcal{I}$, for $k \geq 2$.

Theorem 2.2 ([14, 16]). The following inclusion of monomial ideals holds:

$$
\operatorname{in}_{\prec}\left(\mathcal{I}^{\{k\}}\right) \subseteq\left(\operatorname{in}_{\prec}(\mathcal{I})\right)^{\{k\}} .
$$

Definition 1. If equality holds in (2.3), then $\prec$ is said to be $k$-delightful for the ideal $\mathcal{I}$. It is said to be delightful for $\mathcal{I}$ if it is $k$-delightful for $\mathcal{I}$, for every $k \geq 2$.

We are interested in recovering the initial ideal $\operatorname{in}_{\prec}\left(\mathcal{I}^{\{k\}}\right)$ with respect to non-delightful term orders. Define $\mathcal{J} \subseteq K\left[x_{0}, \ldots, x_{r}\right]$ to be the ideal given by the ideal $\mathcal{I} \cap$ $K\left[x_{0}, \ldots, x_{\delta}\right], 1 \leq \delta \leq r-1$, obtained from $\mathcal{I}$ by elimination plus the ideal generated by the eliminated variables $x_{\delta+1}, \ldots, x_{r}$. Then we have the following inclusion of monomial ideals

$$
\operatorname{in}_{\prec}\left(\mathcal{I}^{\{k\}}\right) \subseteq \operatorname{in}_{\prec}\left(\mathcal{J}^{\{k\}}\right)
$$

for any term order $\prec$. Putting together (2.3) and (2.4) we obtain the following

Proposition 2.5. In the notation as above,

$$
\operatorname{in}_{\prec}\left(\mathcal{I}^{\{k\}}\right) \subseteq\left(\operatorname{in}_{\prec}(\mathcal{I})\right)^{\{k\}} \cap \operatorname{in}_{\prec}\left(\mathcal{J}^{\{k\}}\right) .
$$

If $\operatorname{in}_{\prec}\left(\mathcal{J}^{\{k\}}\right)$ does not contain $\left(\operatorname{in}_{\prec}(\mathcal{I})\right)^{\{k\}}$, then Proposition 2.5 provides a correction of the monomial ideal $\left(\operatorname{in}_{\prec}(\mathcal{I})\right)^{\{k\}}$ to get a smaller ideal which is closer to the initial ideal $\operatorname{in}_{\prec}\left(\mathcal{I}^{\{k\}}\right)$. We will go more into the details of this for toric ideals in Section 3.3.

For projective toric varieties the operation of taking the initial ideal with respect to a term order such that $\operatorname{in}_{\prec}(\mathcal{I})$ is generated by squarefree monomials leads to the notion of triangulations of polytopes. Let $\pi_{D}$ be a toric degeneration of a toric variety $X$ of dimension $n$ to a union of $\mathbb{P}^{n}$ 's. Any subset of $D$ of $k$ pairwise skew $\mathbb{P}^{n}$ 's, i.e. $k(n+1)$ vertices of $D$ that are the vertices of $k$ disjoint tetrahedra of $D$, will span a linear subspace 
of $\mathbb{P}^{r}$ of dimension $k n+k-1$. A subset of this type is said to be a skew $k$-set. We denote by $N_{k}(D)$ the set of such skew $k$-sets and by $\bar{\nu}_{k}(D)$ its cardinality, see $[5,16]$. Sturmfels and Sullivant proved that $\bar{\nu}_{k}(D)$ is a lower bound for the number $\nu_{k}(X)$ for toric varieties.

Theorem 2.6 ([16, Theorem 5.4]). If there exists a toric degeneration $\pi_{D}$ of $X$ to a union of $\mathbb{P}^{n}$ 's such that $\bar{\nu}_{k}(D) \geq 1$, then $\operatorname{Sec}_{k}(X)$ has the expected dimension and $\nu_{k}(X)$ is bounded below by the number of skew $k$-sets:

$$
\nu_{k}(X) \geq \bar{\nu}_{k}(D) \text {. }
$$

Proof. Let $\mathcal{I}$ be the ideal of $X$ and let $\mathcal{I}_{0}$ be the ideal of the central fiber $X_{0}$ with respect to the toric degeneration $\pi_{D}$. The simplicial complex of $\mathcal{I}_{0}$ is $D$. Let $D^{\{k\}}$ be the simplicial complex of $\mathcal{I}_{0}^{\{k\}}$ : the simplices in $D^{\{k\}}$ are the unions of $k$ simplices in $D$, see [16, Remark 2.9]. Notice that the simplices of $D^{\{k\}}$ of maximal dimension are the skew $k$ sets and the subspaces they span sit in the flat limit of $\operatorname{Sec}_{k}(X)$. Therefore, if there exists at least one skew $k$-set in $D$, then $\operatorname{Sec}_{k}(X)$ has the expected dimension $k n+k-1$.

Notice that different skew $k$-sets could span the same subspace $\pi$ of $\mathbb{P}^{r}$ and that for the general point of $\pi$ there is a unique subspace of dimension $k-1$ meeting the $k$ planes each in a point, for each skew $k$-set spanning $\pi$. The variety described by $D^{\{k\}}$ is the reduced union of the coordinate subspaces in $\mathbb{P}^{r}$ given by the skew $k$-sets. Furthermore, the limit of the $k$-secant variety of $X$ contains the variety defined by $\mathcal{I}_{0}^{\{k\}}$ by Theorem 2.2. This concludes the proof.

In [16] the authors conjectured that if equality holds in (2.7), then the term order corresponding to the triangulation $D$ is $k$-delightful. We will call such degenerations $k$-delightful, see also [5, Section 5].

Now, fix $k=2$ and consider the examples in Figure 4.

$D$
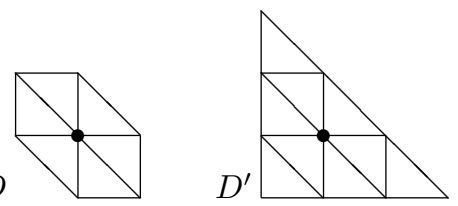

Figure 4. Non-2-delightful triangulations.

The first picture represents a triangulation $D$ of the hexagon $P^{1}(6,6,1)$, i.e., a degeneration of the smooth del Pezzo surface $X \subseteq \mathbb{P}^{6}$ to a union of six planes intersecting at a point. Since $\bar{\nu}_{2}(D)=0$ and $\nu_{2}(X)=3, D$ is not 2-delightful. The second one represents a triangulation of the polytope $P^{1}(3,9,3)$ defining the Veronese surface $X^{\prime}$ in $\mathbb{P}^{9}: \bar{\nu}_{2}\left(D^{\prime}\right)=12$ and $\nu_{2}\left(X^{\prime}\right)=15$ hence $D^{\prime}$ is not 2-delightful. Notice that in both cases there is a 2-delightfulness defect equal to 3 . It is natural to wonder if the cause has to be sought in the sextuple central point, marked in the figures, that intuitively prevents the presence of disjoint triangles in the configurations. More generally, how do the singularities of the configuration influence the delightfulness property? This question was asked by Ciliberto, Dumitrescu and Miranda [5]. Our aim is to give an explanation of this phenomenon. In the next section we will propose our results in this direction. 


\section{A lower bound for the number $\nu_{k}, k=2,3$, for toric surfaces}

Let $X$ be a projective toric surface and let $P \subseteq \mathbb{R}^{2}$ be the defining convex lattice polytope. Let $\pi_{D}$ be a planar toric degeneration of $X$ and let $D$ be the corresponding regular unimodular triangulation of $P$. Let $p \in P \cap \mathbb{Z}^{n}$ be a lattice point of $P$ and let $Q^{1}, \ldots, Q^{\delta} \in D$ be the triangles in $D$ covering $p: Q^{1} \cap \cdots \cap Q^{\delta}=\{p\}$. Suppose that the union of the $Q^{i}$ 's is a a convex subpolytope $Q_{p}$ of $P$. Notice that $Q_{p}$ has either $g=0$ if $p$ is a boundary point of $P$, or $g=1$ if $p$ is an interior point (cf. Theorem 1.1 and Theorem 1.2). Write $Q_{p}=P^{g}(l, \delta, m)$ and define $Z_{p}$ to be the projective toric surface of degree $\delta$ defined by $Q_{p}: Z_{p} \subseteq \mathbb{P}^{\delta^{\prime}} \subseteq \mathbb{P}^{r}$, where $\delta^{\prime}=\delta-g+1$.

This section is devoted to the proof of the following result that improves the lower bound for $\nu_{k}$ of Theorem 2.6 for the cases $n=2, k=2,3$.

Theorem 3.1. Let $k \in\{2,3\}$. Let $X=X_{P}$ be a projective toric surface such that $\operatorname{dim}\left(\operatorname{Sec}_{k}(X)\right)=3 k-1$. Let $D$ be any triangulation of $P$. Let $\left\{p_{i}\right\}_{i \in I} \subseteq P \cap \mathbb{Z}^{n}$, $\left\{Q_{p_{i}}\right\}_{i \in I}$ and $\left\{Z_{p_{i}}\right\}_{i \in I}$ be as above. Assume that

(1) $\operatorname{dim} \operatorname{Sec}_{k}\left(Z_{p_{i}}\right)=3 k-1$, for $i \in I$,

(2) there exists a regular subdivision $D_{i}^{1}$ of $P$ that contains $Q_{p_{i}}$ and which is a coarsening of $D$.

Then $D$ is not $k$-delightful. Moreover

$$
\nu_{k}(X) \geq \bar{\nu}_{k}(D)+\sum_{i \in I} \nu_{k}\left(Z_{p_{i}}\right) .
$$

If $p$ is a boundary lattice point, i.e. $Q_{p}$ has $g=0$, we will call it a rational singularity for $D$, while if $p$ is an interior point, i.e. $Q_{p}$ has $g=1$, we will say that $p$ is an elliptic singularity for $D$. In Table 1 and Table 2 all these singularities are classified.

Remark 3.3. This result also holds for $k \geq 4$. However, since the expected dimension of $\operatorname{Sec}_{k}(X)$ is $\min \{3 k-1, r\}$ and $\operatorname{dim}\left(\operatorname{Sec}_{k}\left(Z_{p}\right)\right)<\operatorname{dim}\left(\operatorname{Sec}_{k}(X)\right)$, for any $Z_{p}$ as in Table 1 or Table 2 , then none of the rational and elliptic singularities gives a contribution to the computation of $\nu_{k}(X)$.

\subsection{Proof of Theorem 3.1.}

3.1.1 The case $\boldsymbol{k}=2$. Let $X=X_{P}$ be a projective toric surface with $\operatorname{dim}(\operatorname{Sec}(X))=$ 5. Let $\pi_{D}$ be a planar toric degeneration of $X$ and let $p$ be a rational or elliptic singularity for $D$. We will prove that the flat limit of the secant variety of $X$ has a 5dimensional component of degree $\nu_{2}\left(Z_{p}\right)$. For this reason, we will assume that $\delta^{\prime} \geq 5$ so that $\operatorname{dim}\left(\operatorname{Sec}_{2}\left(Z_{p}\right)\right)=5$ (cf. Section 2.1). Furthermore we assume that there is a lifting function $F_{D^{1}}$ over an intermediate regular subdivision $D^{1}$ of $P$, that contains $Q$ and other polytopes obtained as union of triangles of $D$. The existence of such an $F_{D^{1}}$ will be discussed in Section 3.2. The regular subdivision $D^{1}$ defines a degeneration $\pi_{D^{1}}$ of $X$ to a reducible surface that has $Z_{p}$ as component. Moreover, call $\pi_{D^{2}}$ the degeneration of the central fiber of $\pi_{D^{1}}$ to $X_{0}$. 
Proposition 3.4. Keeping the same setting as above, if there exists in $D$ a singularity $p$ as in Table 1 or Table 2 and if there exists a regular subdivision $D^{1}$ of $P$ as above, then

$$
\nu_{2}(X) \geq \bar{\nu}_{2}(D)+\nu_{2}\left(Z_{p}\right) .
$$

Proof. Consider first the degeneration $\pi_{D^{1}}$ of $X$. Denote by $X_{t}^{1}$ the fibers of $D^{1}: X_{t}^{1} \cong$ $X$, for $t \neq 0$, while $X_{0}^{1}$ is the reduced union of the toric surfaces corresponding to the family $D^{1}$. The 2-secant variety of $Z_{p}$ and all the joins between components of $X_{0}^{1}$ sit in

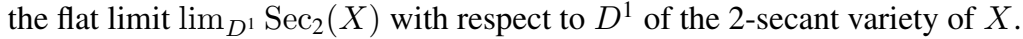

Consider now $\pi_{D^{2}}$ which has as general fiber $X_{s}^{2} \cong X_{0}^{1}, s \neq 0$, and as central fiber the reduced union of planes $X_{0}^{2} \cong X_{0}$. The flat limit, with respect to $D^{2}$, of $\lim _{D^{1}} \operatorname{Sec}_{2}(X)$, namely $\lim _{D} \operatorname{Sec}_{2}(X)$, contains as components the flat limits, with respect to $D^{2}$, of all components of $\lim _{D^{1}} \operatorname{Sec}_{2}(X)$. In particular $\lim _{D} \operatorname{Sec}_{2}(X)$ contains $\lim _{D^{2}} \operatorname{Sec}_{2}\left(Z_{p}\right)$, which is a 5-dimensional component of degree $\nu_{2}\left(Z_{p}\right)$. Furthermore it contains the flat limit via $D^{2}$ of all the joins between components of $X_{s}^{2}, s \neq 0$, including the $\mathbb{P}^{5}$ 's spanned by the skew 2 -sets $N_{2}(D)$.

The contributions in terms of degree given by $\lim _{D^{1}} \operatorname{Sec}_{2}(X)$ and $N_{2}(D)$ can be summed up. Indeed none of the $\mathbb{P}^{5}$ 's spanned by the skew 2 -sets are contained in the limit $\lim _{D^{2}} \operatorname{Sec}_{2}(Z)$.

If $\left\{p_{i}\right\}_{i \in I}$ are singularities of $D$ all of them satisfying the conditions of Proposition 3.4, then the contributions given by $\nu_{2}\left(Z_{p_{1}}\right)$ 's do not interfere with each other. To see this, let us decompose the degeneration $D$ by taking subdivisions $D_{i}^{1}$ and $D_{i}^{2}$, for each $i$. The flat limit of the secant variety of $Z_{p_{i}}$ with respect to $D_{i}^{2}$ sits in the flat limit of the secant variety of $X$ with respect to $D$, for every $i$, by Proposition 3.4. Furthermore, let $\mathbb{P}_{i} \subseteq \mathbb{P}^{r}$ be the projective subspace where $Z_{p_{i}}$, $\operatorname{Sec}_{k}\left(Z_{p_{i}}\right)$ and their limits live, namely the space whose coordinates are given by the lattice points of $Q_{p_{i}}$. Notice that $\operatorname{dim}\left(\mathbb{P}_{i} \cap \mathbb{P}_{j}\right) \leq 3$, for all $i \neq j$. Indeed there are at most two coplanar triangles with vertices at two distinct points $p_{i}, p_{j}$. Since $\left(\lim _{D_{i}^{2}} \operatorname{Sec}_{2}\left(Z_{p_{i}}\right)\right) \cap\left(\lim _{D_{i}^{2}} \operatorname{Sec}_{2}\left(Z_{p_{i}}\right)\right) \subseteq \mathbb{P}_{i} \cap \mathbb{P}_{j}$, then they have no common 5-dimensional component, for all $i, j \in I, i \neq j$, and none of them contains any $\mathbb{P}^{5}$ spanned by elements of $N_{2}(D)$. Hence the respective degrees sum up to $\bar{\nu}_{2}(D)$. This proves Theorem 3.1 for the case $k=2$.

Example 3.6. Consider the embedding $X \subseteq \mathbb{P}^{11}$ of $\mathbb{P}^{1} \times \mathbb{P}^{1}$ via the line bundle $\mathcal{O}(2,3)$. We have $\nu_{2}(X)=\operatorname{deg}\left(\operatorname{Sec}_{2}(X)\right)=35$, see for example [7, Corollary 1.6]. Consider the triangulations $D$ and $D^{\prime}$ of the corresponding rectangle displayed in Figure 5.

$D$ :

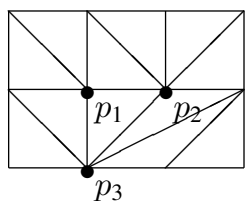

$D^{\prime}$ :

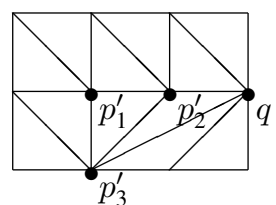

Figure 5. Triangulations of a rectangle.

In the first example the sum of the number of skew 2-sets and of the contributions of the singularities restores the 2-secant degree, $\bar{\nu}_{2}(D)+\nu_{2}\left(X_{p_{1}}\right)+\nu_{2}\left(X_{p_{2}}\right)+\nu_{2}\left(X_{p_{3}}\right)=35$, 
while in the second example we have $\bar{\nu}_{2}\left(D^{\prime}\right)+\nu_{2}\left(X_{p_{1}^{\prime}}\right)+\nu_{2}\left(X_{p_{2}^{\prime}}\right)+\nu_{2}\left(X_{p_{3}^{\prime}}\right)=33$. Notice that in $D^{\prime}$ there is a lattice boundary point $q$ which is covered by five triangles. It causes an obstruction to the presence of skew 2-sets, hence it may affect the delightfulness of $D^{\prime}$, but the polygon given as union of the five triangles is not convex and our argument does not apply to this case.

3.1.2 The case $\boldsymbol{k}=3$. Let $X=X_{P}$ be a toric surface such that $\operatorname{dim}\left(\operatorname{Sec}_{2}(X)\right)=8$. Let $D$ be any regular unimodular triangulation of $P$. There is only one class of rational singularity we are interested in. Indeed the only $g=0$ toric surface with 3 -secant variety of dimension 8 and for which there exists a toric degeneration to a union of planes intersecting at a single point is the rational normal scroll $S(2, \delta-2) \subseteq \mathbb{P}^{\delta+1}$, corresponding to the trapezium $P^{0}(4, \delta, \delta-2)$, with $\delta \geq 7$ (see Table 1, last row). On the other hand, the only $g=1$ toric surfaces we have to consider are the Veronese surface $V_{3} \subseteq \mathbb{P}^{9}$ defined by $P^{1}(3,9,3)$ and the del Pezzo surface $X_{8} \subseteq \mathbb{P}^{8}$ defined by $P^{1}(4,8,3)$. Indeed in all remaining cases (see Table 2 ) the 3 -secant varieties have dimension less than 8 , so they do not contribute to the 2 -secant degree computation.

Proposition 3.7. In the same notation as above, let $p$ be a multiple point such that the corresponding polytope $Q_{p}$ is either $P^{0}(4, \delta, \delta-2)$, with $\delta \geq 7$, or $P^{1}(3,9,3)$, or $P^{1}(4,8,3)$. Assume furthermore that there exists an intermediate regular subdivision $D^{1}$ of $P$ containing $Q_{p}$ and coarsening $D$. Then

$$
\nu_{3}(X) \geq \bar{\nu}_{3}(D)+\nu_{3}\left(Z_{p}\right) .
$$

Proof. It is easy to see that $\operatorname{Sec}_{3}\left(Z_{p}\right)$ and the joins $J\left(Y_{i}, J\left(Y_{j}, Y_{l}\right)\right)$, where $Y_{i}, Y_{j}, Y_{l}$ are components of $\lim _{D^{1}} X$, are in the flat $\operatorname{limit}_{D^{1}} \operatorname{Sec}_{3}(X)$.

Then, let $\pi_{D^{2}}$ be the degeneration of the central fiber of $\pi_{D^{1}}$ to the union of planes given by the triangulation $D$. The $\mathbb{P}^{8}$ 's spanned by the skew 3 -sets of $D$ and the limit $\lim _{D^{2}} \operatorname{Sec}_{3}(Z)$ are 8-dimensional components of the limit of $\operatorname{Sec}_{3}(X)$ with respect to $D$. Furthermore, the contributions $\bar{\nu}_{3}(D)$ and $\nu_{3}\left(Z_{p}\right)$ do not interfere with each other, because they do not have common full dimensional components.

If there are singularities $\left\{p_{i}\right\}_{i \in I}$ for $D$ satisfying the hypotheses of Proposition 3.7, arguing as for the case $k=2$, we get Inequality (3.2) for $k=3$.

\subsection{On the existence of an intermediate regular subdivision of $P$ containing $Q$ and} coarsening $D$. Let us construct first a partition of $P$ containing $Q$, triangles and convex polytopes given as union of triangles of $D$ using the following algorithm. Let $L_{1} \ldots, L_{l}$ be the edges of $Q$. For $i=1, \ldots, l$, define $S_{i}$ to be the minimal convex union of triangles of $D$ such that $S_{i} \cap Q=L_{i}$. Now, let $L_{i, 1}, \ldots, L_{i, l_{i}}$ be the edges of $S_{i}$, for $i \in\{1, \ldots, l\}$ and define $S_{i, j}$ as the minimal convex union of triangles of $D$ such that $S_{i, j} \cap S_{i}=L_{i, j}$, $i=1, \ldots, l, j=1, \ldots, l_{i}$. Repeat the process until a family of polytopes whose union is $P$ is constructed. Notice that this new subdivision $D^{1}$ is a coarsening of the original regular subdivision $D$. It gives rise to a toric degeneration whether it is possible to flatten the lifting function $F_{D}$ over $Q$, the $S_{i}$ 's, the $S_{i, j}$ 's, etc., by rescaling it in such a way that the resulting piecewise linear function $F_{D^{1}}$ is strictly convex over $P$. 
Example 3.9. Consider the triangulation $D$ of the rectangle in Example 3.6 and define the following functions.

$$
\begin{array}{rcccrcccrccc}
12 & 6 & 2 & 0 & 12 & 6 & 2 & 0 & 12 & 6 & 4 & 2 \\
F_{D}: 12 & 5 & 0 & 2 & F_{D_{1}^{1}}: 12 & 6 & 0 & 2, & F_{D_{2}^{1}}: 12 & 6 & 4 & 2 \\
14 & 6 & 10 & 16 & 6 & 10 & 16 & 6 & 10 & 16
\end{array} .
$$

The first one defines the regular subdivision $D$ of the rectangle, while $F_{D_{1}^{1}}, F_{D_{2}^{1}}$ are modifications of $F_{D}$ that give rise to the subdivisions $D_{1}^{1}, D_{2}^{1}$ of the rectangle in Figure 6.
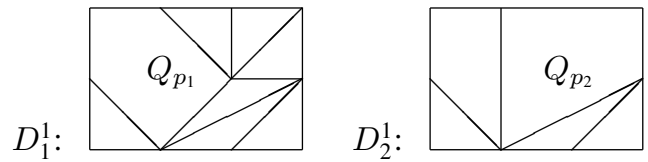

Figure 6. Examples of intermediate regular subdivisions.

3.3 Computing the initial ideal of the secant ideal. Let $\mathcal{I}_{X}$ and $\mathcal{I}_{Z_{p_{i}}}$ denote the toric ideals of $P$ and $Q_{p_{i}}, i \in I$, respectively. Let $\mathcal{J}_{Z_{p_{i}}}$ be the ideal defined by the generators of $\mathcal{I}_{Z_{p_{i}}}$ and by all variables not corresponding to lattice points of $Q_{p_{i}}$. As the referee suggested, the following holds.

Theorem 3.10. In the notation as above, then

$$
\operatorname{in}_{\prec}\left(\mathcal{I}_{X}^{\{k\}}\right) \subseteq\left(\operatorname{in}_{\prec}\left(\mathcal{I}_{X}\right)\right)^{\{k\}} \cap \bigcap_{i} \operatorname{in}_{\prec}\left(\mathcal{J}_{Z_{p_{i}}}^{\{k\}}\right) .
$$

Proof. It follows from Proposition 2.5.

The proof of Theorem 3.1 implies that if the number $\nu_{k}\left(Z_{p_{i}}\right)$ is non-zero, then the ideal $\mathcal{J}_{Z_{p_{i}}}^{\{k\}}$ gives an effective correction to the secant ideal of the initial ideal of $\mathcal{I}$. Indeed, since the ideals $\mathcal{J}_{Z_{p_{i}}}^{\{k\}}, k \in\{2,3\}$ are known (see for example [12, Section 5.2] for an overview), one can easily compute the initial ideal with respect to the triangulation $D_{\mid Q_{p_{i}}}$ of $Q_{p_{i}}$.

Example 3.11. Consider the regular unimodular triangulation $D$ of the rectangle in Example 3.6. The coordinates $x_{0}, \ldots, x_{11}$ of $\mathbb{P}^{11}$ correspond to the lattice points of the polytope. Label them from left to right, top to bottom as follows.

$\begin{array}{llll}x_{0} & x_{1} & x_{2} & x_{3} \\ x_{4} & x_{5} & x_{6} & x_{7} \\ x_{8} & x_{9} & x_{10} & x_{11}\end{array}$

The initial ideal of $X$ with respect to $D$ is the edge ideal consisting of all squarefree quadratic monomials corresponding to non-edges of $D: \operatorname{in}_{\prec}\left(\mathcal{I}_{X}\right)=\left\langle x_{0} x_{2}, x_{0} x_{3}, \ldots\right.$, 
$\left.x_{9} x_{11}\right\rangle$. The ideal $\left(\operatorname{in}_{\prec}\left(\mathcal{I}_{X}\right)\right)^{\{2\}}$ is generated by the squarefree monomials of degree at least 3 corresponding to subsets of lattice points of $P$ that are pairwise not on a edge of $D:\left(\operatorname{in}_{\prec}\left(\mathcal{I}_{X}\right)\right)^{\{2\}}=\left\langle x_{0} x_{2} x_{7}, \ldots, x_{6} x_{8} x_{11}, x_{0} x_{2} x_{7} x_{11}, \ldots, x_{3} x_{5} x_{8} x_{11}\right\rangle$, see [16, Theorem 3.2].

The 2-secant variety of $Z_{p_{1}} \subseteq \mathbb{P}^{5}$ fills up the space given by the six lattice points of $Q_{p_{1}}$, then $\operatorname{in}_{\prec}\left(\mathcal{J}_{Z_{p_{1}}}^{\{2\}}\right)=\mathcal{J}_{Z_{p_{1}}}^{\{2\}}=\left\langle x_{2}, x_{3}, x_{7}, x_{8}, x_{10}, x_{11}\right\rangle$. The equation of the 2 -secant variety of $Z_{p_{2}}$ in $\mathbb{P}^{6}$ is known:

$$
\operatorname{det}\left|\begin{array}{lll}
x_{1} & x_{2} & x_{5} \\
x_{2} & x_{3} & x_{6} \\
x_{5} & x_{6} & x_{9}
\end{array}\right|=0
$$

We can easily check that the restriction to $K\left[x_{1}, x_{2}, x_{3}, x_{5}, x_{6}, x_{7}, x_{9}\right]$ of the term order on $K\left[x_{0}, \ldots, x_{11}\right]$ corresponding to $D$ selects the main diagonal product as the leading term of this determinant, thus we get $\operatorname{in}_{\prec}\left(\mathcal{J}_{Z_{p_{2}}}^{\{2\}}\right)=\left\langle x_{1} x_{3} x_{9}, x_{0}, x_{4}, x_{8}, x_{10}, x_{11}\right\rangle$. The equation of the 2 -secant variety of the rational normal scroll $Z_{p_{3}}$ is known as well:

$$
\operatorname{det}\left|\begin{array}{ccc}
x_{8} & x_{4} & x_{5} \\
x_{9} & x_{5} & x_{6} \\
x_{10} & x_{6} & x_{7}
\end{array}\right|=0
$$

Thus $\operatorname{in}_{\prec}\left(\mathcal{J}_{Z_{p_{3}}}^{\{2\}}\right)=\left\langle x_{j_{1}} x_{j_{2}} x_{j_{3}}, x_{0}, x_{1}, x_{2}, x_{3}, x_{10}, x_{11}\right\rangle$, where $\left(j_{1}, j_{2}, j_{3}\right)$ equals either $(8,5,7)$ or $(4,6,10)$. The ambiguity is due to the fact that two different term orders may lead to the same triangulation, so we have to operate a choice between the two possible leading terms. Nevertheless, in both cases the intersection $\left(\operatorname{in}_{\prec}(\mathcal{I})\right)^{\{2\}} \cap \bigcap_{i=1}^{3} \operatorname{in}_{\prec}\left(\mathcal{J}_{Z_{p_{i}}}^{\{2\}}\right)$ contains the initial ideal in $\prec\left(\mathcal{I}^{\{2\}}\right)$ and has the same degree and dimension.

\section{Delightful triangulations of $g=0$ polygons}

In this section we classify all $g=0$ convex lattice polytopes that admit delightful triangulations. As a preliminary remark, notice that a necessary condition for the degeneration to be 2-delightful is that it contains no lattice point as in Table 1 in its configuration. We will see that the triangulations with this property turn out to be delightful.

The triangle $P^{0}(3,4,2)$ admits two regular unimodular triangulations, up to lattice equivalence, see Figure 7 . They are both delightful, in fact in both cases $\operatorname{in}_{\prec}\left(\mathcal{I}^{\{2\}}\right)=$
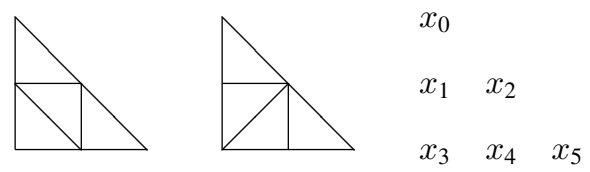

Figure 7. Regular unimodular triangulations of $P^{0}(3,4,2)$. 


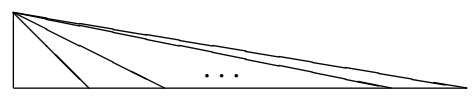

$$
\begin{array}{llllll}
x_{0} & & & & & \\
x_{1} & x_{2} & x_{3} & \ldots & x_{i} & x_{i+1}
\end{array}
$$

Figure 8. Regular unimodular triangulation of $P^{0}(3, i, i)$.

$$
\left(\operatorname{in}_{\prec}(\mathcal{I})\right)^{\{2\}}=\left\langle x_{0} x_{3} x_{5}\right\rangle \text { and } \operatorname{in}_{\prec}\left(\mathcal{I}^{\{k\}}\right)=\left(\operatorname{in}_{\prec}(\mathcal{I})\right)^{\{k\}}=\langle 0\rangle, k \geq 3 .
$$

The unique regular unimodular triangulation of the triangle $P^{0}(3, i, i)$ (cf. Figure 8) is delightful. Indeed the ideal $\operatorname{in}_{\prec}\left(\mathcal{I}^{\{k\}}\right)=\left(\operatorname{in}_{\prec}(\mathcal{I})\right)^{\{k\}}$ is generated by all squarefree monomials of degree $k+1$ in $x_{1}, \ldots, x_{i+1}$ if $i \geq 2 k$ and it is null if $i<2 k$.

The most interesting case involves trapezia.

Theorem 4.1. The trapezium $P^{0}(4,2 \delta+i, \delta+i), \delta \geq 1$, admits delightful triangulations if and only if $0 \leq i \leq 3$.

The unique delightful triangulations of $P^{0}(4,2 \delta+i, \delta+i)$, up to lattice equivalence, are the ones represented in Figure 9.

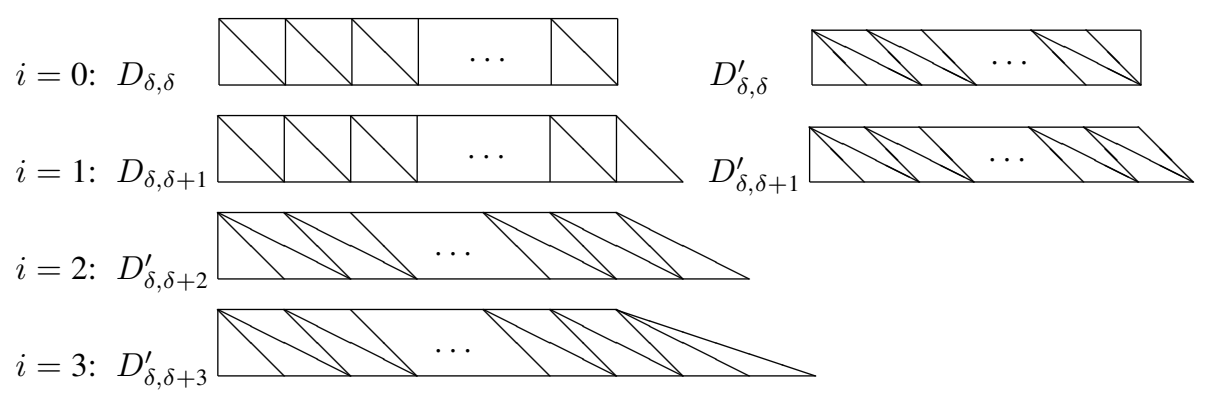

Figure 9. Delightful triangulations of $g=0$ polytopes.

Recall that the rational normal scroll $X$ defined by $P:=P^{0}(4,2 \delta+i, \delta+i), \delta \geq 1$, $0 \leq i \leq 3$ is non- $k$-defective and minimal $k$-secant degree, for any $k$ such that $3 k-1 \leq$ $2 \delta+i+1$ (cf. Section 2.1.1). Therefore, in order to prove that a triangulation $D$ of $P$ is $k$-delightful, it is enough to verify that the number $\bar{\nu}_{k}(D)$ equals the $k$-secant degree whether $\operatorname{dim}\left(\operatorname{Sec}_{k}(X)\right)=3 k-1$. Otherwise it is enough to show that $\left(\operatorname{in}_{\prec}(\mathcal{I})\right)^{\{k\}}=\langle 0\rangle$, where $\prec$ is a term order corresponding to $D$.

It is useful to have a recursive formula for $\bar{\nu}_{k}(D)$. Set $d:=2 \delta+i$. Let $D$ be a regular unimodular triangulation of $P$ as in Figure 9. Call $T$ the last triangle of $D$ and define $P_{1} \subseteq$ $P$ to be the subpolytope of $P$ such that $P \backslash P_{1}=T$. We have $P_{1}=P^{0}\left(4,2 \delta_{1}+i_{1}, \delta+i_{1}\right)$, $0 \leq i_{1} \leq 3$, with Area $\left(P_{1}\right)=d-1=2 \delta_{1}+i_{1}$ for some $\delta_{1}, i_{1}$. Notice that the triangulation $D_{1}:=D_{\mid P_{1}}$ of $P_{1}$ is regular and lattice equivalent to one of the configurations in Figure 9. Define moreover $D_{2}:=\left\{T_{2} \in D_{1}: T_{2} \cap T=\emptyset\right\} \subseteq D_{1} \subseteq D$ : it is given by those triangles of $D$ which do not intersect $T$. One can see that $D_{2}$ is a regular triangulation, as in Figure 9, of a polytope $P_{2}=P^{0}\left(4,2 \delta_{2}+i_{2}, \delta+i_{2}\right) \subseteq P_{1}, 0 \leq i_{2} \leq 3$, for some 
$\delta_{2}, i_{2}$. We can now split $N_{k}(D)$ in two sets: the skew $k$-sets contained in $D_{1}$ on one side and those involving $T$ on the other side: $N_{k}(D)=N_{k}\left(D_{1}\right) \cup\left\{\left(T,\left(T_{2,1}, \ldots, T_{2, k-1}\right)\right)\right.$ : $\left.\left(T_{2,1}, \ldots, T_{2, k-1}\right) \in N_{k-1}\left(D_{2}\right)\right\}$. Thus we get the formula:

$$
\bar{\nu}_{k}(D)=\bar{\nu}_{k}\left(D_{1}\right)+\bar{\nu}_{k-1}\left(D_{2}\right)
$$

Lemma 4.3. In the above notation, $D$ is $k$-delightful if and only if $D_{1}$ is $k$-delightful and $D_{2}$ is $(k-1)$-delightful.

Proof. Since $D_{2}$ contains at most $d-3$ triangles, then $\bar{\nu}_{k-1}\left(D_{2}\right) \leq\left(\begin{array}{c}(d-3)-2(k-2) \\ k-1\end{array}\right)$. Exploiting Formula (4.2) we get $\bar{\nu}_{k}(D) \leq\left(\begin{array}{c}(d-1)-2(k-1) \\ k\end{array}\right)+\left(\begin{array}{c}(d-3)-2(k-2) \\ k-1\end{array}\right)=\left(\begin{array}{c}d-2(k-1) \\ k\end{array}\right)$. Since the number on the right equals $\nu_{k}\left(X_{P}\right)$ the assertion follows.

This argument allows to use induction on $d$ and $k$ to prove that the degenerations depicted in Figure 9 are $k$-delightful, for $k$ such that $3 k-1 \leq d+1$.

Proof of Theorem 4.1. First of all notice that every regular unimodular triangulation of $P=P^{0}(4,2 \delta+i, \delta+i)$ different from the ones in Figure 4.1 contains a rational singularity. Assume that $D$ is as in Figure 9. It is easy to prove that $D$ is 2-delightful by induction on $d$ using Formula (4.2).

To prove $k$-delightfulness for $k \geq 3$, assume that $(k-1)$-delightfulness holds for any degeneration of $P$ as in Figure 9. Define $d_{0}=d_{0}(k):=3 k-2$. If $d<d_{0}$ then $k$-delightfulness trivially holds. For $d=d_{0}$ we have $\bar{\nu}_{k}(D)=\bar{\nu}_{k}\left(D_{1}\right)+\bar{\nu}_{k-1}\left(D_{2}\right)=$ $0+1=1$. For the case $d>d_{0}$, assume that $k$-delightfulness holds for degree $\leq d-1$ and apply induction to conclude.

In [16, Proposition 5.8] Sturmfels and Sullivant proved that if a delightful term order exists for a rational normal scroll $S\left(\delta_{1}, \ldots, \delta_{n}\right)$ of dimension $n$, then $\delta_{j} \in\{m, m+1, m+$ $2, m+3\}$ for some $m$. In particular they showed that the six configurations of Figure 9 are the only possible delightful triangulations of the trapezium $P^{0}(4,2 \delta+1, \delta+1)$. Indeed they are the unique triangulations whose associated bipartite graphs do not posses certain induced subgraphs, which correspond to the rational singularities in Table 1. Moreover the proofs of [16, Theorem 5.9 and Proposition 5.11] show that all the triangulations down the first column of Figure 9, namely $D_{\delta, \delta}, D_{\delta, \delta+1}, D_{\delta, \delta+2}^{\prime}$ and $D_{\delta, \delta+3}^{\prime}$, are delightful.

In Theorem 4.1 we gave a slightly different proof of this and moreover we showed that $D_{\delta, \delta}^{\prime}$ and $D_{\delta, \delta+1}^{\prime}$ are delightful as well.

In [16] the authors described the delightful term orders corresponding to the triangulations $D_{\delta, \delta}, D_{\delta, \delta+1}, D_{\delta, \delta+2}^{\prime}$ and $D_{\delta, \delta+3}^{\prime}$ and computed the initial ideals of the secant ideals. For the sake of completeness we describe here the initial ideal of the $k$-secant of the ideal $\mathcal{I}$ of $S(\delta, \delta)$ and $S(\delta, \delta+1)$ with respect to $D_{\delta, \delta}^{\prime}$ and $D_{\delta, \delta+1}^{\prime}$ respectively. Consider first the rectangle $P^{0}(4,2 \delta, \delta)$ and label the lattice points of the bottom basis from $(1,0)$ to $(1, \delta)$ and the lattice points of the top basis from $(2,0)$ to $(2, \delta)$. The lexicographic term order corresponding to $D_{\delta, \delta}^{\prime}$ is defined by the following rule:

$$
\begin{array}{r}
x_{10} \succ x_{11} \succ x_{20} \succ x_{12} \succ \cdots \succ x_{1 j} \succ x_{2, j-1} \succ x_{1, j+1} \succ \cdots \succ x_{1 \delta} \succ x_{2, \delta-1} \succ x_{2 \delta} . \\
\text { Brought to you by | Loughborough University } \\
\text { Authenticated } \\
\text { Download Date | 6/2/17 2:44 PM }
\end{array}
$$


The initial ideal $\operatorname{in}_{\prec}\left(\mathcal{I}^{\{k\}}\right)=\left(\operatorname{in}_{\prec}(\mathcal{I})\right)^{\{k\}}$ is generated by the squarefree monomial of degree $k+1$ corresponding to $(k+1)$-sets of lattice points of $P^{0}(4,2 \delta, \delta)$ that are pairwise not on a edge of $D_{\delta, \delta}^{\prime}$, namely the diagonal products of the $(k+1) \times(k+1)$ minors of

$$
\left(\begin{array}{cccccc}
x_{10} & \cdots & x_{1, \delta-k} & x_{20} & \cdots & x_{2, \delta-k} \\
\vdots & \ddots & \vdots & \vdots & \ddots & \vdots \\
x_{1 k} & \cdots & x_{1 \delta} & x_{2 k} & \cdots & x_{2 \delta}
\end{array}\right),
$$

if $\delta \geq k+1$, otherwise it is null. For the triangulation $D_{\delta, \delta+1}^{\prime}$ of $P^{0}(4,2 \delta+1, \delta+1)$ the description is similar and left to the reader.

\section{Tables}

In Table 1 and Table 2 the triangulations of polytopes $Q_{p}$ are collected. The point $p$ is marked in the pictures. The degree of the associated toric surface $Z_{p}$ is written in the second column, while the numbers $\nu_{2}\left(Z_{p}\right)$ and $\nu_{3}\left(Z_{p}\right)$ are collected in the third and fourth column, if $\operatorname{dim}\left(\operatorname{Sec}_{k}\left(Z_{p}\right)\right)=3 k-1$, otherwise we write " /".

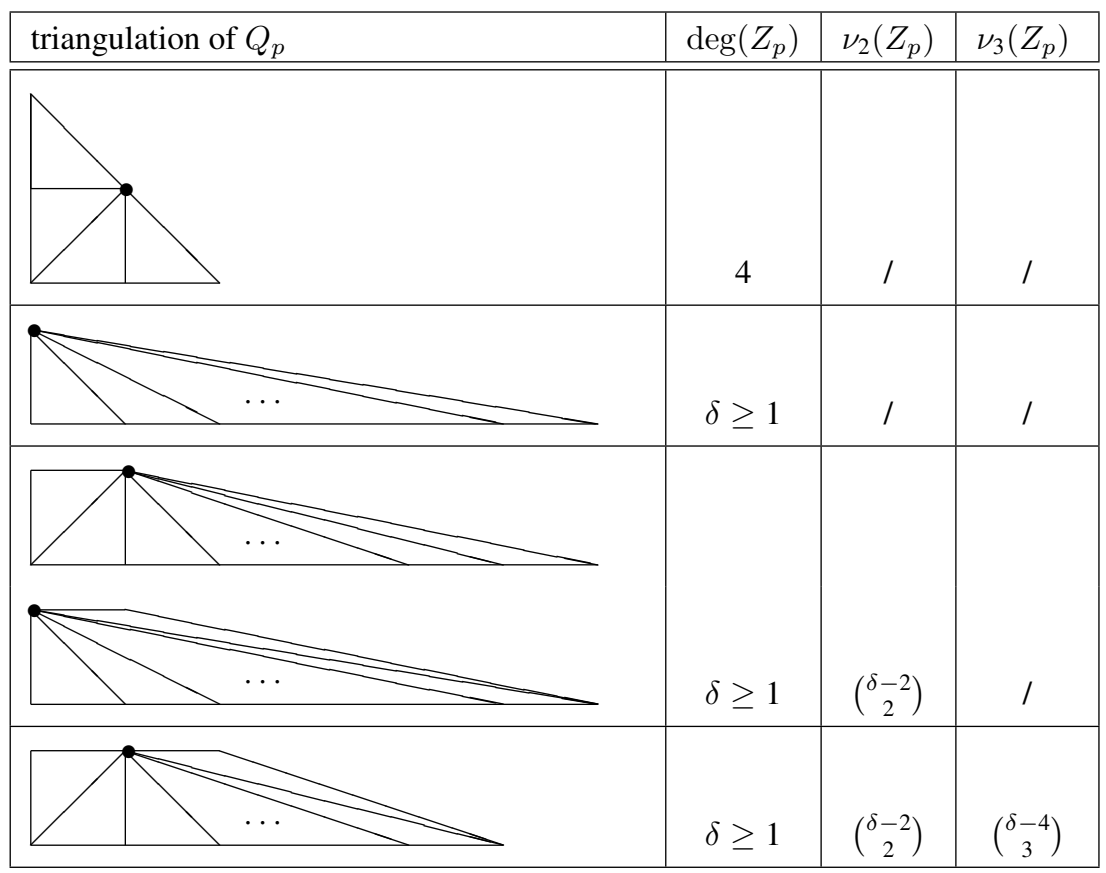

Table 1. Rational singularities. 


\begin{tabular}{|l|c|c|c|}
\hline triangulation of $Q_{p}$ & $\operatorname{deg}\left(Z_{p}\right)$ & $\nu_{2}\left(Z_{p}\right)$ & $\nu_{3}\left(Z_{p}\right)$ \\
\hline
\end{tabular}

Table 2. Elliptic singularities.

\section{References}

[1] M. L. Catalano-Johnson, The possible dimensions of the higher secant varieties. Amer. J. Math. 118 (1996), 355-361. MR1385282 (97a:14058) Zbl 0871.14043

[2] M. V. Catalisano, A. V. Geramita, A. Gimigliano, On the ideals of secant varieties to certain rational varieties. J. Algebra 319 (2008), 1913-1931. MR2392585 (2009g:14068) Zbl 1142.14035

[3] L. Chiantini, C. Ciliberto, Weakly defective varieties. Trans. Amer. Math. Soc. 354 (2002), 151-178. MR1859030 (2003b:14063) Zbl 1045.14022 
[4] L. Chiantini, C. Ciliberto, On the concept of $k$-secant order of a variety. J. London Math. Soc. (2) 73 (2006), 436-454. MR2225496 (2007k:14110) Zbl 1101.14067

[5] C. Ciliberto, O. Dumitrescu, R. Miranda, Degenerations of the Veronese and applications. Bull. Belg. Math. Soc. Simon Stevin 16 (2009), 771-798. MR2574360 (2011a:14007) Zbl 1183.14018

[6] C. Ciliberto, F. Russo, Varieties with minimal secant degree and linear systems of maximal dimension on surfaces. Adv. Math. 200 (2006), 1-50. MR2199628 (2007d:14097) Zbl 1086.14043

[7] D. Cox, J. Sidman, Secant varieties of toric varieties. J. Pure Appl. Algebra 209 (2007), 651669. MR2298847 (2008i:14077) Zbl 1115.14045

[8] J. A. De Loera, J. Rambau, F. Santos, Triangulations, volume 25 of Algorithms and Computation in Mathematics. Springer 2010. MR2743368 (2011j:52037) Zbl 1207.52002

[9] W. Fulton, Introduction to toric varieties, volume 131 of Annals of Mathematics Studies. Princeton Univ. Press 1993. MR1234037 (94g:14028) Zbl 0813.14039

[10] S. Hu, Semistable degeneration of toric varieties and their hypersurfaces. Comm. Anal. Geom. 14 (2006), 59-89. MR2230570 (2007k:14101) Zbl 1115.14030

[11] R. J. Koelman, The number of moduli of families of curves on toric surfaces. Thesis, Nijmegen, 1991.

[12] E. Postinghel, Degenerations and applications: polynomial interpolation and secant degree. $\mathrm{PhD}$ thesis, Università degli Studi Roma Tre, 2010.

[13] S. Rabinowitz, A census of convex lattice polygons with at most one interior lattice point. Ars Combin. 28 (1989), 83-96. MR1039134 (91f:52019) Zbl 0704.52006

[14] A. Simis, B. Ulrich, On the ideal of an embedded join. J. Algebra 226 (2000), 1-14. MR1749874 (2001f:13031) Zbl 1034.14026

[15] B. Sturmfels, Gröbner bases and convex polytopes, volume 8 of University Lecture Series. Amer. Math. Soc. 1996. MR1363949 (97b:13034) Zbl 0856.13020

[16] B. Sturmfels, S. Sullivant, Combinatorial secant varieties. Pure Appl. Math. Q. 2 (2006), 867891. MR2252121 (2007h:14082) Zbl 1107.14045

Received 3 January, 2011; revised 31 August, 2011

E. Postinghel, Centre of Mathematics for Applications, University of Oslo, P. O. Box 1053 Blindern, 0316 Oslo, Norway

Email: elisa.postinghel@cma.uio.no, elisa.postinghel@gmail.com 